\title{
Water Management in Micro Direct Methanol Fuel Cells: Using Inte- grated Passive Micromixer
}

\author{
Chun-You Lin*, ${ }^{1}$, Hsien-Chun Meng ${ }^{2}$ and C. $\mathrm{Fu}^{1}$ \\ ${ }^{1}$ Institute of NanoEngineering and MicroSystems, National Tsing-Hua University; ${ }^{2}$ Micro Base Technology Corp., \\ No.101, Kuang-fu Rd., Sec.II, Hsinchu 300, Taiwan, R.O.C
}

\begin{abstract}
In a DMFC (direct methanol fuel cell), water management is a critical issue for enhancing DMFC power performance and lifetime. However, the methanol-water mixing tank for water management is difficult to be miniaturized and integrated into a micro-DMFC. This paper proposes an in-fuel cell micromixer, the self-vortical micromixer, which belongs to a passive micromixer, to perform methanol and water mixing inside a micro-DMFC.

Experimental results demonstrate the design, fabrication, and testing results of the self-vortical micromixer integrated with the micro-DMFC, showing that the self-vortical micromixer has high fluidic mixing performance and can be operated in the micro-DMFC as a methanol-water mixing tank for water management of the micro-DMFC. The power performance testing results demonstrate that the single cell of micro-DMFC, with the included self-vortical micromixer, exhibits a maximum power density of $21.27 \mathrm{~mW} / \mathrm{cm}^{2}$.
\end{abstract}

Keywords: Micro-DMFC, water management, microfluidic micromixer.

\section{INTRODUCTION}

Microfluidic micromixers are extremely essential components used in numerous micro-chip microfluidic systems for biochemistry analysis, drug discovery, and nucleic acid sequencing and synthesis [1]. For example, a microfluidic biochip uses a micromixer in the fluidic mixing procedure of small volume biological samples and reaction reagents [2]. In addition, a microfluidic micromixer is possible to be used in a micro-DMFC.

A fuel cell is an electrochemical power generator that converts the chemical energy of a chemical reaction to electricity. The electrochemical reaction equations of a DMFC are listed as below:

$$
\begin{gathered}
\text { Anode reaction: } \mathrm{CH}_{3} \mathrm{OH}+\mathrm{H}_{2} \mathrm{O} \longrightarrow \mathrm{CO}_{2}+6 \mathrm{H}^{+}+6 \mathrm{e}^{-} \\
\text {Cathode reaction: } 6 \mathrm{H}^{+}+3 / 2 \mathrm{O}_{2}+6 \mathrm{e}^{-} \longrightarrow 3 \mathrm{H}_{2} \mathrm{O} \\
\text { Overall reaction: } \mathrm{CH}_{3} \mathrm{OH}+3 / 2 \mathrm{O}_{2} \longrightarrow \mathrm{CO}_{2}+2 \mathrm{H}_{2} \mathrm{O}
\end{gathered}
$$

Methanol with the specific concentration as a reductant is fed to the anode for oxidation reaction on the anode of a DMFC. Oxygen from the air as an oxidant is supplied to the cathode for reduction reaction on the cathode of a DMFC. Therefore, the power performance of a DMFC is highly sensitive not only to the concentration of providing methanol to the anode, but also to the flux of providing oxygen to the cathode.

A DMFC consists of an electrolyte membrane (proton exchange membrane, or PEM), anode components, (comprising an anode catalyst layer), an anode gas diffusion layer,

*Address correspondence to this author at the Institute of NanoEngineering and MicroSystems, National Tsing-Hua University, No.101, Kuang-fu Rd., Sec.II, Hsinchu 300, Taiwan, R.O.C; Tel: +886-3-571-5131 ext. 3996; Fax: +886-3-572-8023; E-mail: frankchunyoulin@gmail.com a gasket, an anode plate, and a flow field plate, as well as cathode components, (comprising a cathode catalyst layer), a cathode gas diffusion layer, a gasket and a cathode plate. A PEM supports hydrogen protons when migrating from the anode components to the cathode components. Anode reactions generate electrons that migrate from the anode plate to the cathode plate. Connecting the anode plate to the cathode plate can generate electricity.

A DMFC has higher power density and relatively higher energy-conversion efficiency than a lithium ion battery does [3]. A large-scale DMFC is able to be miniaturized to become a micro-DMFC as a favorable candidate for the increasing energy demand of portable electronics, such as cellular phones, laptop computers, and PDAs.

Unfortunately for DMFC commercialization, water management concerns remain, such as cathode water flooding, methanol feeding, methanol crossover, and water balance; all of which must be resolved. In a DMFC, cathode water flooding affects water balance, and methanol feeding affects methanol supplies, including not only the consumption of the newly supplied methanol fed from the outside, but also the usage of the recirculation methanol originating from the anode.

Nevertheless, in a micro-DMFC, water management is more difficult than that in a large-scale DMFC. For water management in a DMFC, many patents provide ideas, such as mixing chambers [4], methanol concentration sensors [5], hydrophilic threads [6], valves [7], water recirculation systems [8], and liquid pumps [9].

In a DMFC, the cathode water-flooding phenomenon is caused by the fact that the excess water, such as the byproduct of cathode reduction reaction, does not drain away and remains in the cathode. Water management is able to balance 
water inside a DMFC more effectively in preventing the cathode water-flooding phenomenon from occurring.

Excess water remaining in the cathode can obstruct outside air from being supplied to the cathode catalyst; therefore, the excess water causes a DMFC to lose power performance. Considerable research has been conducted on the water management of a DMFC to achieve higher power performance and lifetime.

In Fig. (1) demonstrates the concept for water management in a micro-DMFC, using an integrated passive micromixer as a methanol-water mixing tank. Firstly, to perform water management in preventing the cathode waterflooding phenomenon, the water remaining in the cathode must be drained away. Secondly, to re-use the recirculated methanol coming from the anode and to maintain the specific concentration of methanol feeding into the anode, the large-scale DMFC design has an external methanol-water

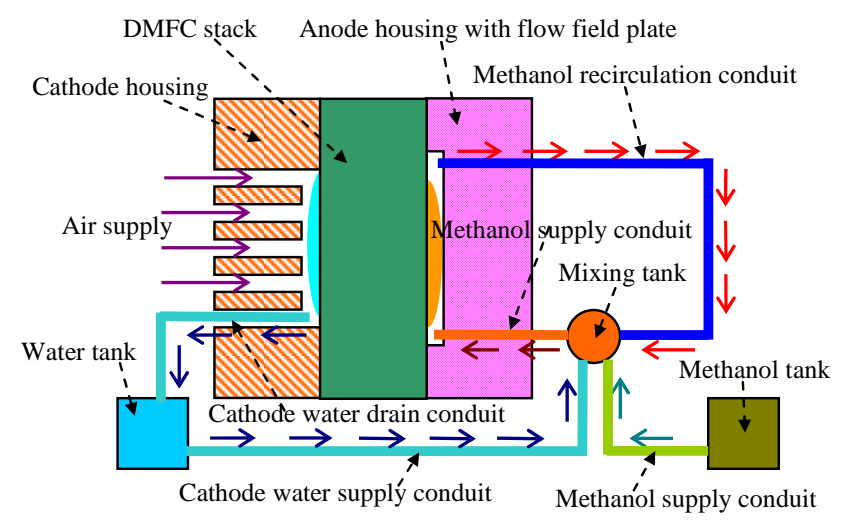

(a)

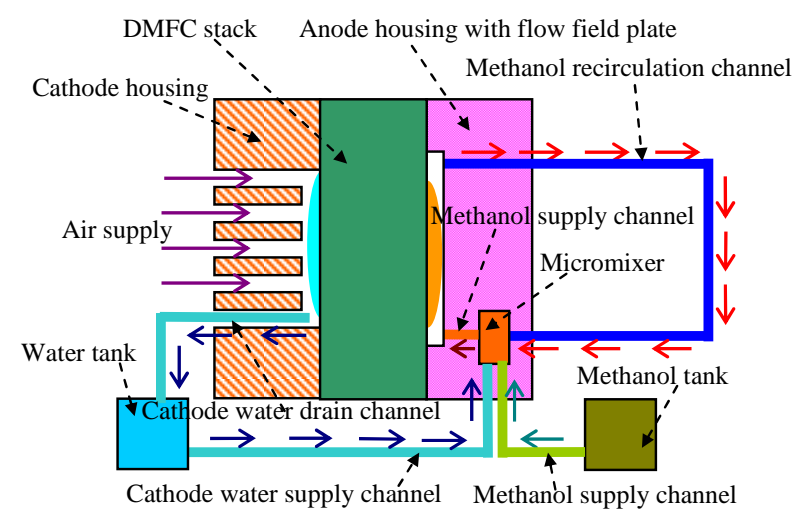

(b)

Fig. (1). The schematic diagram of the water management for a large-scale DMFC and a micro-DMFC designed in this study. (a) For maintaining the specific concentration of methanol and well water management, the large-scale DMFC design has an external methanol-water mixing tank for methanol and water mixing, in turn feeding into the anode of the large-scale DMFC. (b) For miniaturizing a DMFC and maintaining well water management, the selfvortical micromixer, as an internal mixing tank, is integrated into a micro-DMFC. The DMFC stack is composed of an anode plate, a gasket (anode side), an anode gas diffusion layer, an anode catalyst layer, a PEM, a cathode catalyst layer, a cathode gas diffusion layer, a gasket (cathode side), and a cathode plate. mixing tank for methanol and water mixing, in turn feeding into the anode of the large-scale DMFC, as shown in Fig. (1a). However, the methanol-water mixing tank may not be able to perform mixing efficiently; moreover, the methanolwater mixing tank is difficult to be miniaturized and integrated inside a micro-DMFC. For miniaturizing a DMFC and managing water effectively, as shown in Fig. (1b), this study developed a new passive micromixer, called a selfvortical micromixer, as a methanol-water mixing tank. Additionally, this paper proposes the micro-DMFC with an internal mixing function of the developed new passive micromixer and demonstrate its power performance.

\section{EXPERIMENTAL}

\subsection{Micromixer and micro-DMFC Design}

Micromixers can be categorized into passive micromixers and active micromixers. Active micromixers mix fluids by either actuating moving parts or using external forces, such as pressure disturbances, electrical fields, and magnetic and acoustic vibrations, to achieve mixing effects [10-15]. Passive micromixers use complicated shapes and structures along microchannels via complicated micromachining processes, and usually require mixing channels with considerable lengths and novel designs to enhance geometric stirring [1621].

Fig. (2a) and (b) show the schematic diagram of the microfluidic mixing principle of the self-vortical micromixer developed in this study. In the self-vortical micromixer, two fluids are separately injected into two inlet reservoirs and separately flow into two inlet channels simultaneously; the two fluids are then able to generate the self-vortical mixing phenomenon in the circular mixing chamber. The mixed fluid mixture of the two fluids is able to flow into the mixing channel and achieve complete mixing.

The self-vortical micromixer is composed of an upper flow field plate and a lower flow field plate, each with a thickness of $2 \mathrm{~mm}$, as shown in Fig. (2c). The upper flow field plate contains two inlet channels, both with a width of 500 microns and a depth of 150 microns; one circular mixing chamber with a diameter of 2000 microns and a depth of 150 microns; and two inlet reservoirs, both with a diameter of 5000 microns and a depth of $20 \mathrm{~mm}$. The lower flow field plate contains one serpentine micro-channel with a width of 500 microns and a depth of 150 microns; one mixing channel with a width of 500 microns; and one outlet reservoir with a diameter of 5000 microns and a depth of $20 \mathrm{~mm}$.

The self-vortical micromixer, consisting of the upper flow field plate and the lower flow field plate, is designed to be integrated into the micro-DMFC developed in this study, with a length of $35 \mathrm{~mm}$, a width of $35 \mathrm{~mm}$, and a thickness of $7 \mathrm{~mm}$.

\subsection{Micromixer and micro-DMFC Fabrication}

The self-vortical micromixer developed in this study consists of an upper flow field plate and a lower flow field plate. We used polydimethylsiloxane (PDMS) material to fabricate both the upper flow field plate and the lower flow field plate using the PDMS replication molding method [22]. The PDMS-manufactured upper flow field plate has the micro feature patterns and involves two inlet channels, one circular 


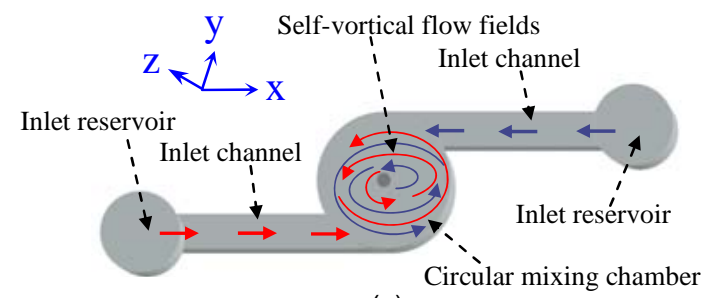

(a)

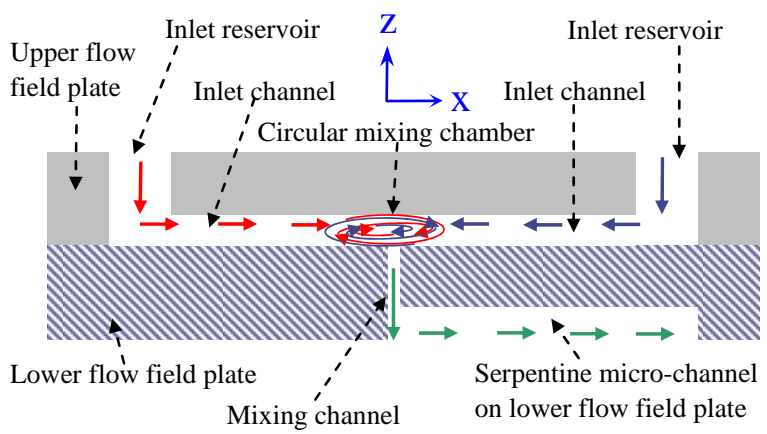

(b)

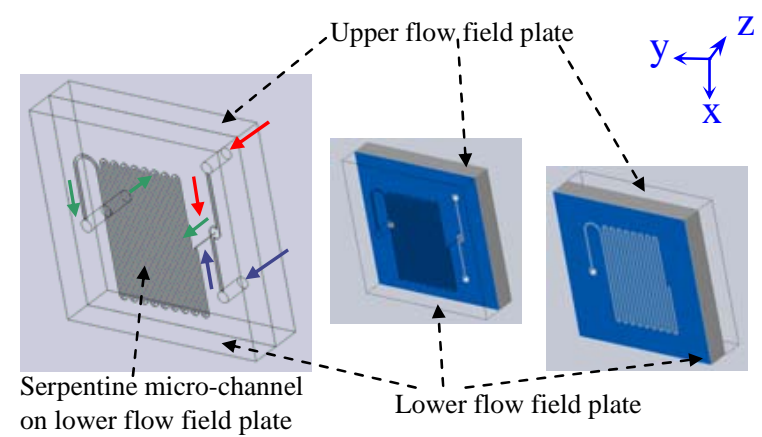

(c)

$\leftarrow$ Fluid $\mathrm{A} \leftarrow$ Fluid $\mathrm{B}$

$\leftarrow$ Fluid mixture of fluid A and fluid B

Fig. (2). The microfluidic mixing principle and design of the selfvortical micromixer. (a) The microfluidic mixing principle is shown in the top-view of the self-vortical micromixer. (b) The microfluidic mixing principle is shown in the cross-section view of the self-vortical micromixer. (c) The designed self-vortical micromixer is integrated into the serpentine micro-channel on lower flow field plate of the micro-DMFC.

mixing chamber, and two inlet reservoirs; conversely, the PDMS-manufactured lower flow field plate has the micro feature patterns and includes one serpentine micro-channel and one outlet reservoir. Firstly, the aforementioned micro feature patterns on replication molds were fabricated using a thick-film photoresist photolithography process, using a chrome photo mask. Secondly, A PDMS pre-polymer was poured on the replication mold and allowed to harden after a baking process; we then peeled the hardened PDMS from the replication mold. Using the aforementioned PDMS replication molding method, we separately fabricated the PDMSmanufactured upper flow field plate and the PDMSmanufactured lower flow field plate. Finally, we used PDMS oxygen plasma treatment for bonding of the PDMS- manufactured upper flow field plate and the PDMSmanufactured lower flow field plate to prevent leakage of water and methanol coming from the micro-channel.

As shown in Fig. (3), we developed the micro-DMFC composed of the following parts: a PMMA plate (anode side), a PDMS-manufactured upper flow field plate, a PDMS-manufactured lower flow field plate, an anode plate, a gasket (anode side), a membrane electrode assembly (including an anode gas diffusion layer, an anode catalyst layer, a PEM, a cathode catalyst layer, and a cathode gas diffusion layer), a gasket (cathode side), a cathode plate, and a PMMA plate (cathode side). The membrane electrode assembly was purchased from Johnson Matthey PLC (United Kingdom).

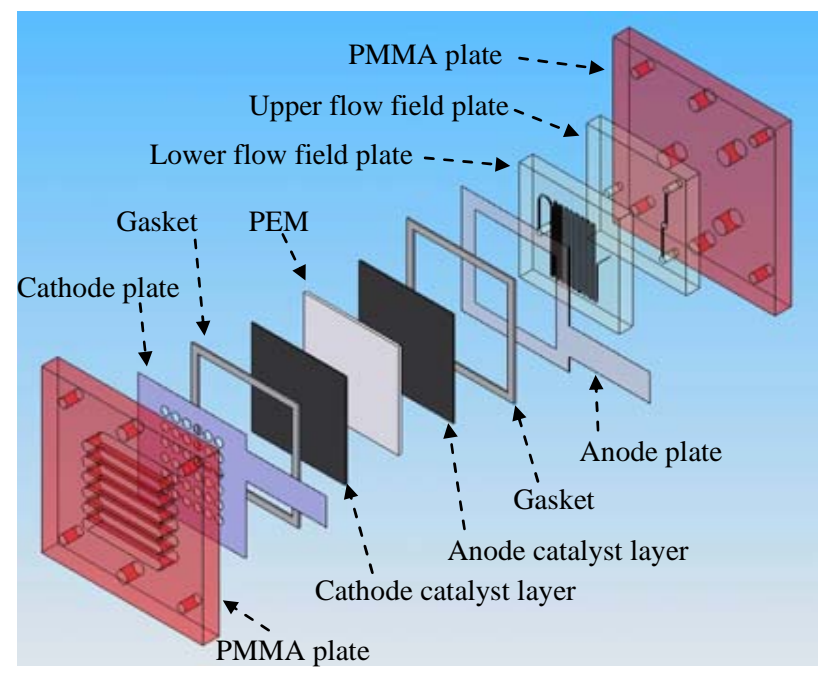

Fig. (3). The assembly picture of the micro-DMFC integrated with the self-vortical micromixer developed in this study. The microDMFC is composed of the following parts: a PMMA plate (anode side), a PDMS-manufactured upper flow field plate, a PDMSmanufactured lower flow field plate, an anode plate, a gasket (anode side), a membrane electrode assembly (including an anode gas diffusion layer, an anode catalyst layer, a PEM, cathode catalyst layer, and a cathode gas diffusion layer), a gasket (cathode side), a cathode plate, and a PMMA plate (cathode side). The PMMA plate (anode side) and the PMMA plate (cathode side) are bound together by using screws.

Fig. (4) shows the photograph of the micro-DMFC developed in this study. The stack of micro-DMFC was composed of an aluminum-manufactured anode plate, a gasket (anode side), an anode catalyst layer, a PEM, a cathode catalyst layer, a gasket (cathode side), and an aluminummanufactured cathode plate. Both the aluminummanufactured anode plate and the aluminum-manufactured cathode plate were fabricated by aluminum material laser micromachining.

\subsection{Micromixer Testing}

As shown in Fig. (5a), we demonstrate the fluidic mixing performance of the self-vortical micromixer. Simultaneously, a blue food dye fluid and a yellow food dye fluid were separately injected into two inlet channels using two pumps with identical flow rates of $2.4 \mathrm{~mL} / \mathrm{min}$; the dyes then flowed into the circular mixing chamber to generate swirly 


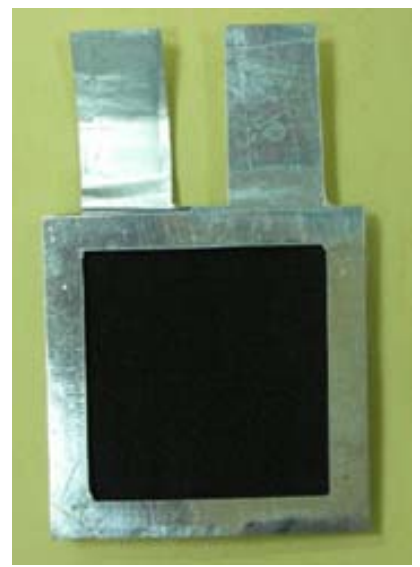

(c)

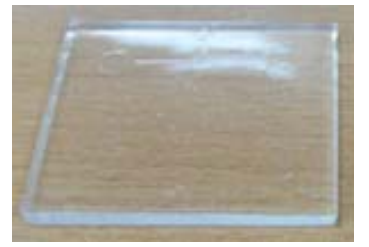

(a)

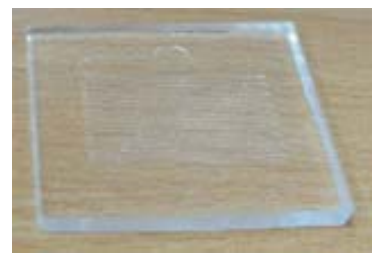

(b)

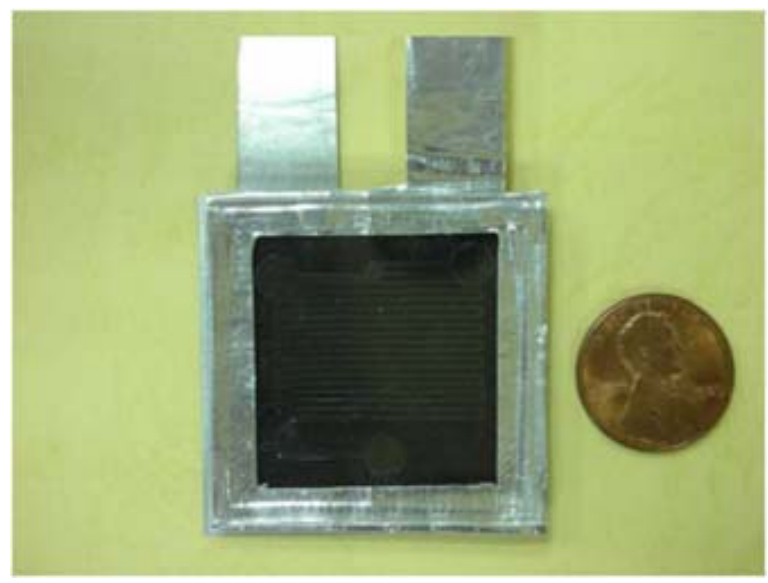

(d)
Fig. (4). The photographs of the micro-DMFC developed in this study. (a) and (b) show the PDMS-manufactured upper flow field plate and the PDMS-manufactured lower flow field plate, respectively; both were composed of PDMS material using PDMS replication molding method. (c) The stack of micro-DMFC was composed of an aluminum-manufactured anode plate, a gasket (anode side), an anode catalyst layer, a PEM, a cathode catalyst layer, a gasket (cathode side), and an aluminum-manufactured cathode plate. (d) The micro-DMFC developed in this study was compared with a penny coin.

flow fields and further generated self-vortical flow fields. The blue food dye fluid and the yellow food dye fluid were mixed to become a green fluid downstream of the mixing channel by the self-vortical flow fields.

The commercial computational fluid dynamics (CFD) package software, CFD-ACE+ (CFD Research Corporation), was used to create a $3 \mathrm{D}$ geometry of the self-vortical micromixer with the assumptions of no-slip boundary condition on walls to demonstrate this mixing effect. Fig. (5b) demonstrates the CFD simulation results that show the self-vortical mixing phenomenon in the self-vortical micromixer. The flow velocity profiles and the flow streamlines in the circular mixing chamber are generated while the water fluids simultaneously flow into the two inlet channels at flow rates of 2.4
$\mathrm{mL} / \mathrm{min}$. The directions of the vectors of the flows represent the directions of flow velocities.

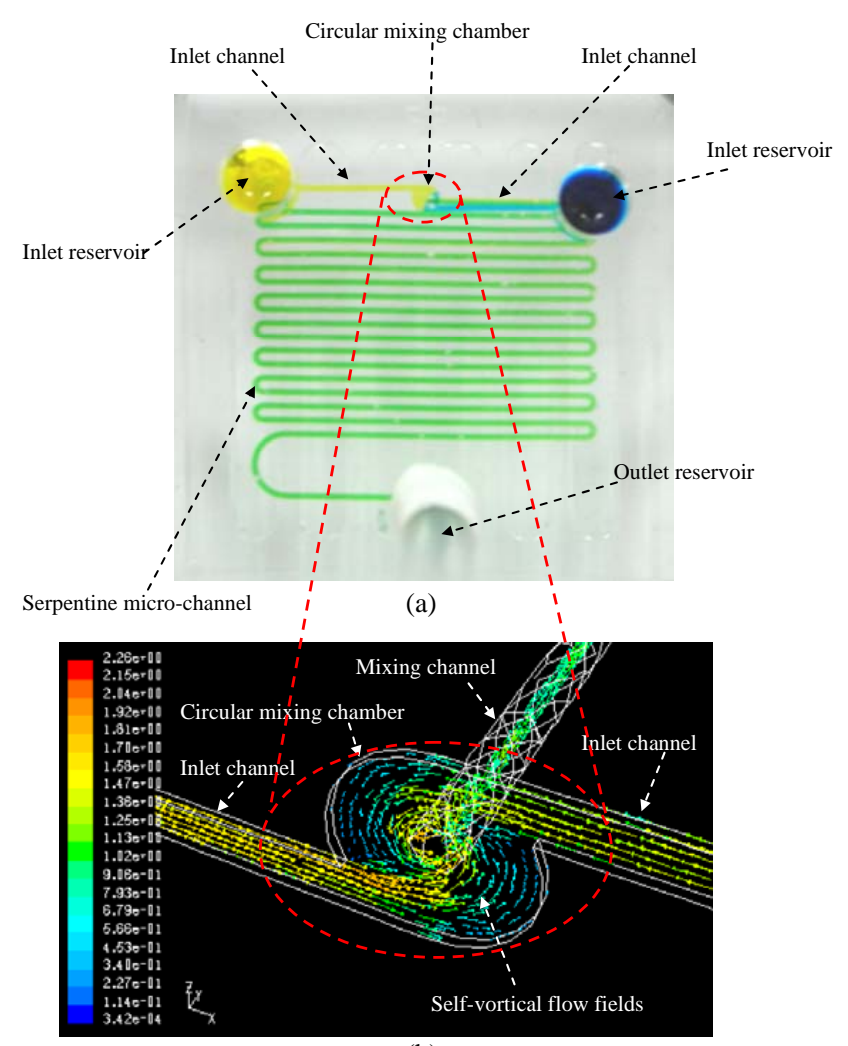

(b)

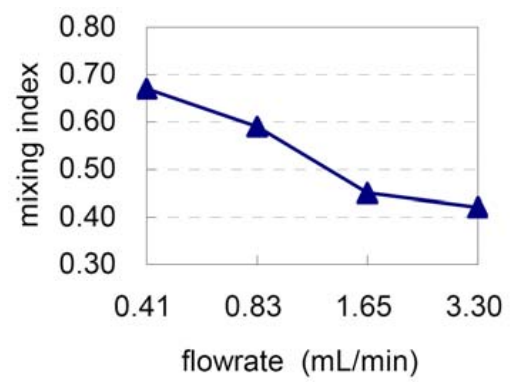

(c)

Fig. (5). The mixing test and CFD simulation results of the selfvortical micromixer. (a) Simultaneously, a blue food dye fluid and a yellow food dye fluid were separately injected into two inlet channels using two pumps with identical flow rates of $2.4 \mathrm{~mL} / \mathrm{min}$; the dyes were then flowed into the circular mixing chamber to generate swirly flow fields and further generated self-vortical flow fields. The blue food dye fluid and the yellow food dye fluid were mixed to become a green fluid downstream of the mixing channel by the self-vortical flow fields. (b) CFD simulation results show the self-vortical mixing phenomenon in the self-vortical micromixer. The flow velocity profiles and the flow streamlines in the circular mixing chamber are generated while the water fluids simultaneously flow into the two inlet channels at flow rates of $2.4 \mathrm{~mL} / \mathrm{min}$. The directions of the vectors of the flows represent the directions of flow velocities. (c) The testing results of mixing index for two different color dye fluids mixed in the circular mixing chamber of the self-vortical micromixer. The lower mixing index indicates a higher mixing efficiency. . 
As shown in Fig. (5c), we characterized the mixing efficiency for the self-vortical micromixer using the definition of the mixing index [23]. The mixing indexes were calculated according to the grayscale values of the pictures of two different color dye fluids mixed downstream of the mixing channel of the self-vortical micromixer, and the blue food dye fluid and the yellow food dye fluid could be completely mixed to become a green fluid mixture.

\subsection{Micro-DMFC Testing}

A methanol solution with a concentration of 9.0 wt. \% and a deionized water solution were prepared to test the power performance of the micro-DMFC. Simultaneously, two pumps with identical flow rates of $2.4 \mathrm{~mL} / \mathrm{min}$ were used to deliver separately the $9.0 \mathrm{wt}$. \% methanol solution and the deionized water solution into one of the two inlet reservoirs of the self-vortical micromixer inside the microDMFC. After fluidic mixing by the self-vortical micromixer, the mixed fluid mixture of the $9.0 \mathrm{wt}$. \% methanol solution and the deionized water solution had a concentration of 4.5 wt. \%. The mixed fluid mixture was transported to the serpentine micro-channel (area was $2.45 \mathrm{~cm}^{2}$ ) on the PDMSmanufactured lower flow field plate of the micro-DMFC; the mixture then flowed into the anode components of the micro-DMFC. During the specific concentration of methanol feeding, the cathode components of the micro-DMFC were exposed to the ambient air for oxygen supplied by natural air convection.

A fuel cell load test system developed in this study was used to measure polarization curves at the polarization condition: scan voltage was $600 \mathrm{mV}$ to $0 \mathrm{mV}$, voltage decrease step was $10 \mathrm{mV}$, and load interval time was 1 minute. Fig. (6) demonstrates the polarization curves of current and voltage characteristics from the polarization test of a single cell of micro-DMFC. Fig. (7) presents the power density curve of the power performance of a single cell of micro-DMFC.

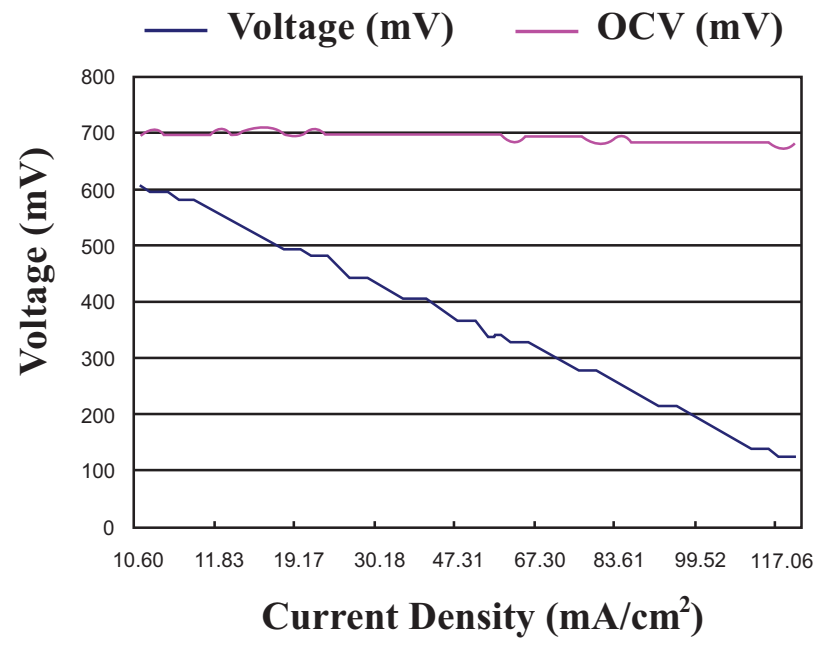

Fig. (6). The polarization curves of the current and voltage characteristics from the polarization test of a single cell of the microDMFC.

\section{RESULTS AND DISCUSSION}

The CFD simulation results demonstrate that the selfvortical mixing phenomenon occurs in the micromixer while the water fluids simultaneously flow into the two inlet channels at flow rates of $2.4 \mathrm{~mL} / \mathrm{min}$. The micromixer test results shows the mixing indexes of the two different color dye fluids mixed at the different inlet-fluid flow rates. The higher inlet-fluid flow rate owns the lower mixing index, and it indicates the micromixer owns higher mixing efficiency when applying at the higher inlet-fluid flow rate. At the inlet-fluid flow rate of $3.30 \mathrm{~mL} / \mathrm{min}$, mixing index 0.42 can be achieved.

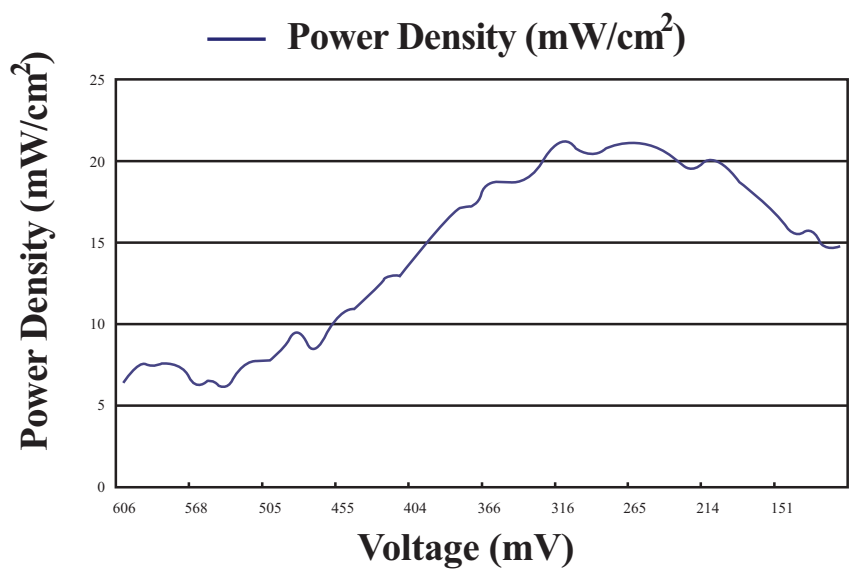

Fig. (7). The chart shows the power density curve of the power performance of a single cell of the micro-DMFC.

According to micro-DMFC test results, the micro-DMFC shows an open circuit voltage $(\mathrm{OCV})$ of $700 \mathrm{mV}$. Due to the activation overpotential is very high in the micro-DMFC, the current density starts from about $10 \mathrm{~mA} / \mathrm{cm}^{2}$. Furthermore, the power performance testing results revealed that the single cell of micro-DMFC exhibits a maximum power density of $21.27 \mathrm{~mW} / \mathrm{cm}^{2}$. Many $\mathrm{CO}_{2}$-gas bubbles generated from the anode of the micro-DMFC causes the performance of the fuel cell to be low, only around $21 \mathrm{~mW} / \mathrm{cm}^{2}$. It must consider a new design for removal of $\mathrm{CO}_{2}$-gas bubbles to enhance the performance of fuel cell.

\section{CONCLUSION}

In this paper, we demonstrate the design, fabrication, and testing results of the self-vortical micromixer integrated with the micro-DMFC developed in this study, showing that the self-vortical micromixer can be operated in the micro-DMFC and can be used as a methanol-water mixing tank for water management of the micro-DMFC. The power performance testing results demonstrate that the micro-DMFC, with included self-vortical micromixer, has a maximum power density of $21.27 \mathrm{~mW} / \mathrm{cm}^{2}$.

\section{ACKNOWLEDGEMENTS}

The authors would like to acknowledge assistance from Micro Base Technology Corp. (www.microbase.com.tw) for providing facilities to test the micro-DMFC.

\section{REFERENCES}

[1] Nguyen, N.-T.; Wu, Z. Micromixers-a review. J. Micromech. Microeng., 2005, 15, R1-R16.

[2] Waters, L. C.; Jacobson, S. C.; Kroutchinina, N.; Khandurina, J.; Foote, R. S.; Ramsey, J. M. Microchip device for cell lysis, multiplex PCR amplification, and electrophoretic sizing. Anal. Chem., 1998, $70,158-162$. 
[3] Lu, G. Q.; Wang, C. Y. Development of micro direct methanol fuel cells for high power applications. J. Power Sources, 2005, 144, 141-145.

[4] Koripella, C. R.; Ooms, W. J.; Wilcox, D. L.; Bostaph, J. W. Direct methanol fuel cell system and method of fabrication. U. S. Patent 6,387,559, 2000 .

[5] Bostaph, J. W.; Koripella, C. R.; Fisher, A. M. Direct methanol fuel cell system including an integrated methanol sensor and method of fabrication. U. S. Patent 6,696,189, 2000.

[6] Neutzler, J.; Bostaph, J. W.; Fisher, A. M. Direct methanol fuel cell including a water management system and method of fabrication. U. S. Patent 6,660,423, 2000

[7] Gottesfeld, S. Methods and apparatuses for a pressure driven fuel cell system. U. S. Patent 6,686,081, 2001.

[8] Bostaph, J. W.; Marshall, D. S. Direct methanol fuel cell including a water recovery and recirculation system and method of fabrication. U. S. Patent 6,727,016, 2001.

[9] Tajima, N.; Sakaue, E.; Matsuoka, K. Fuel Cell. U. S. Patent 7,318,969, 2008.

[10] Volpert, M.; Meinhart, C. D.; Mezic, I.; Dahelh, M. An actively controlled micromixer, Proc. MEMS ASME IMECE, 1999; pp. 483487.

[11] Lee, Y. K.; Deval, J.; Tabeling, P.; Ho, C. M. Chaotic mixing in electrokinetically and pressure driven micro flow, Proc. IEEE MEMS, 2001; pp. 483-486.

[12] Niu, X.; Lee, Y. K. Efficient spatial-temporal chaotic mixing in microchannels. J. Micromech. Microeng., 2003, 13, 454-462.

[13] Oddy, M. H.; Santiago, J. G.; Mikkelsen, J. C. Electrokinetic instability micromixing. Anal. Chem., 2001, 73, 5822-5832.
[14] Lu, L.-H.; Ryu, K. S.; Liu, C. A magnetic microstirrer and array for microfluidic mixing. J. Microelectromech. Syst., 2002, 11, 462469.

[15] Yang, Z.; Goto, H.; Matsumoto, M.; Maeda, R. Active micromixer for microfluidic systems using lead-zirconate-titanate (PZT)generated ultrasonic vibration. Electrophoresis, 2000, 21, 116-119.

[16] Liu, R. H.; Stremler, M. A.; Sharp, K. V.; Olsen, M. G.; Santiago, J. G.; Adrian, R. J.; Aref, H.; Beebe, D. J. Passive mixing in a three-dimensional serpentine microchannel. J. Microelectromech. Syst., 2000, 9, 190-197.

[17] Stroock, A. D.; Dertinger, S. K. W.; Ajdari, A.; Mezic, I.; Stone, H. A.; Whitesides, G. M. Chaotic mixer for microchannels. Science, 2002, 295, 647-651.

[18] Bertsch, A.; Heimgartner, S.; Cousseau, P.; Renaud, P. 3D micromixers-downscaling large scale industrial static mixers, Proc. IEEE MEMS, 2001; pp. 507-510.

[19] Bertsch, A.; Heimgartner, S.; Cousseau, P.; Renaud, P. Static micromixers based on large-scale industrial mixer geometry. Lab Chip, 2001, 1, 56-60.

[20] Kim, D. S.; Lee, I. H.; Kwon, T. H.; Cho, D.-W. A barrier embedded Kenics micromixer. J. Micromech. Microeng., 2004, 14, 1294 1301.

[21] Kim, D. S; Lee, S. W.; Kwon, T. H.; Lee, S. S. A barrier embedded chaotic micromixer. J. Micromech. Microeng., 2004, 14, 798-805.

[22] Part, S.-J.; Kim, J. K.; Park, J.; Chung, S.; Chung, C.; Chang, J. K. Rapid three-dimensional passive rotation micromixer using the breakup process. J. Micromech. Microeng., 2004, 14, 6-14.

[23] Wu, H.-Y.; Liu, C.-H. A novel electrokinetic micromixer. Sens. Actuators A, 2005, 118, 107-115.

(C) Lin et al.; Licensee Bentham Open.

This is an open access article licensed under the terms of the Creative Commons Attribution Non-Commercial License (http://creativecommons.org/licenses/by-nc/3.0/) which permits unrestricted, non-commercial use, distribution and reproduction in any medium, provided the work is properly cited. 